\title{
Interleukin-6 receptor inhibition with tocilizumab induced a selective and substantial increase in plasma IP-10 and MIP-1 $\beta$ in non-ST-elevation myocardial infarction
}

Ola Kleveland, ${ }^{1,2 *}$ Thor Ueland,,${ }^{4,5,6,7}$ Gabor Kunszt, ${ }^{5,10}$ Marte Bratlie, ${ }^{4,5,10}$ Kaspar Broch,,${ }^{7,10}$ Espen Holte, ${ }^{1,2}$ Liv Ryan, ${ }^{3}$ Brage H. Amundsen,,${ }^{1,2}$ Bjørn Bendz, ${ }^{10}$ Svend Aakhus, ${ }^{1,2}$ Terje Espevik, ${ }^{3}$ Tom E. Mollnes, ${ }^{3,6,9,11,12}$ Rune Wiseth,,${ }^{1,2}$ Lars Gullestad,,${ }^{5,7,8,10}$ Pål Aukrust, ${ }^{4,5,6}$ Jan Kristian Damås ${ }^{3}$

${ }^{1}$ Clinic of Cardiology, St. Olavs Hospital, ${ }^{2}$ Department of Circulation and Medical Imaging, ${ }^{3}$ Centre of Molecular Inflammation Research, Department of Cancer Research and Molecular Medicine, Norwegian University of Science and Technology NTNU, Trondheim, ${ }^{4}$ Research Institute of Internal Medicine, Oslo University Hospital Rikshospitalet, ${ }^{5}$ Institute of Clinical Medicine, ${ }^{6}$ K.G. Jebsen Centre of Inflammatory Research, ${ }^{7}$ K.G. Jebsen Cardiac Research Centre, ${ }^{8}$ Centre for Heart Failure Research, University of Oslo, ${ }^{9}$ Department of Immunology,

${ }^{10}$ Department of Cardiology, Oslo University Hospital Rikshospitalet, Oslo, ${ }^{11}$ Research laboratory, Nordland Hospital, Bod $\varnothing$, and ${ }^{12}$ Faculty of Health Sciences, K.G. Jebsen Thrombosis Research and Expertise Center, University of Tromsø, Tromsø, Norway

*Corresponding author: Ola Kleveland Clinic of Cardiology St. Olavs University Hospital P.B. 3250 Sluppen 7006 Trondheim Norway 
E-mail: ola.kleveland@gmail.com

Phone: +47 91300368

Fax: +4772828370

\section{Keywords:}

Tocilizumab

IL-6

Inflammation

Myocardial infarction

IP-10

MIP-1 $\beta$ 


\begin{abstract}
Aim: To evaluate the effect of interleukin-6 inhibition with tocilizumab on the cytokine network in patients with acute non-ST-elevation myocardial infarction (NSTEMI).
\end{abstract}

Methods: 117 patients with acute NSTEMI were randomised to an intravenous infusion of $280 \mathrm{mg}$ tocilizumab or placebo prior to coronary angiography. Blood samples were obtained at baseline, at 6 consecutive timepoints during hospitalisation, and during follow-up. Cytokines $(\mathrm{n}=27)$ were analysed at all timepoints using a multiplex cytokine assay. Results: Using a mixed between-within subjects analysis of variance, we observed a significant $(\mathrm{p}<0.001)$ tocilizumab*time interaction for interferon gamma-inducible protein (IP-10) and macrophage inflammatory protein-1 $\beta$ (MIP-1 $\beta$ ) during hospitalisation, due to significant increases in the tocilizumab group for both IP-10 (median change from baseline during hospitalisation $\left(m_{\Delta}\right)$, placebo: $3(-60) \mathrm{pg} / \mathrm{ml}$ vs tocilizumab: 209 (69) pg/ml) and MIP$1 \beta$ ( $m_{\Delta}$, placebo: $5(-2.1) \mathrm{pg} / \mathrm{ml}$ vs tocilizumab $\left.39(24) \mathrm{pg} / \mathrm{ml}\right)$ compared to placebo, and significant between-group differences in changes from baseline $(\mathrm{p}<0.001)$ at all timepoints. MIP-1 $\beta$ was inversely correlated to troponin $\mathrm{T}(\mathrm{r}=-0.28, \mathrm{p}<0.05)$ and neutrophils $(\mathrm{r}=-0.32$, $\mathrm{p}<0.05)$ in the tocilizumab group during hospitalisation.

Conclusions: Tocilizumab led to a substantial increase in IP-10 and MIP-1 $\beta$ during the acute phase of NSTEMI. This increase was selective, since none of the other cytokines were affected in the tocilizumab group. ClinicalTrials.gov, NCT01491074. 


\section{Introduction}

Inflammation plays a crucial role in atherogenesis both in the chronic phase, but also during plaque rupture with subsequent development of complications like myocardial infarction (MI) and ischemic stroke.[1] MI could again trigger a comprehensive inflammatory response characterised by homing of leukocytes to the infarcted myocardium and upregulation of several cytokines and chemokines.[2] While this complex and timely regulated inflammatory surge seems to be essential for infarct healing and cardiac repair in MI,[2] an exaggerated or protracted inflammatory response may contribute to increased infarct-size, and this could also include adverse inflammatory responses during ischaemiareperfusion (I/R) injury.[3] Thus, modulating inflammation could be an attractive therapeutic target in MI with potentially beneficial effects on plaque rupture, I/R injury and infarct size.

Interleukin-6 (IL-6) is a multifunctional and pro-inflammatory cytokine which is upregulated and appears to hold a central position in the inflammatory response during plaque destabilisation[4] and MI.[5] In MI, elevated levels of IL-6 are associated with infarct size and an adverse prognosis.[5] Furthermore, IL-6 seems to be involved in myocardial I/R injury, $[6,7]$ and increasing levels of IL-6 also predict adverse left ventricular (LV) remodeling after acute MI.[8] In the inflammatory response during MI, IL-6 represents an intermediate cytokine which is strongly induced by apical cytokines such as IL-1 and tumor necrosis factor (TNF),[9] while it is the major inducer of downstream acute phase responses, including hepatic production of C-reactive protein (CRP).[10]

We have recently shown that IL-6 inhibition by a single intravenous administration of $280 \mathrm{mg}$ of the IL-6 receptor (R) antagonist tocilizumab attenuated inflammation and percutaneous coronary intervention (PCI)-related troponin $\mathrm{T}(\mathrm{TnT})$ release in patients with non-ST-elevation MI (NSTEMI).[7] These results could suggest a beneficial effect of IL-6 inhibition on inflammatory mediated I/R injury in these patients. However, cytokines operate 
in complex networks, and the ability of tocilizumab to affect other inflammatory and antiinflammatory mediators with relevance to NSTEMI is at present not known. In this predefined sub-study, we sought to evaluate the effects of IL-6 inhibition on the cytokine network in patients with acute NSTEMI by analysing a wide range of circulating cytokines during hospitalisation and follow-up (i.e. 3 and 6 months post-MI). Furthermore, a dose of $280 \mathrm{mg}$ of tocilizumab provides total IL-6 blockade for 2-3 weeks, [11] and it is important to explore to which extent IL-6 inhibition affects different actors in the cytokine network not only in the acute phase, but also during follow-up. 


\section{Methods}

\section{Study population and design}

This pre-defined sub-study was part of a two-center, randomised, double-blind, placebo-controlled trial investigating the effect of a single dose of the anti-IL-6R antagonist tocilizumab in patients with NSTEMI scheduled for coronary angiography (ClinicalTrials.gov, NCT01491074). The study was performed at Oslo university hospital Rikshospitalet, Oslo, and St. Olavs hospital, Trondheim, Norway. The study was approved by the Regional Committee for Medical and Health Research Ethics of South-Eastern Norway and the Norwegian Medicines Agency, and conducted according to the Helsinki Declaration. All participants provided written, informed consent.

The study design has been reported previously.[7] In short, patients with acute NSTEMI presumed to be caused by coronary artery disease (CAD) were included on the day of scheduled coronary angiography. Major exclusion criteria were other clinically significant heart disease, clinical instability, medication or diseases affecting inflammation, and any condition that could interfere with protocol adherence. After baseline blood sampling, patients received a 1 hour infusion containing either $280 \mathrm{mg}$ tocilizumab or matching placebo (100 $\mathrm{mL}$ 0.9\% NaCl) intravenously, prior to coronary angiography. After coronary angiography and percutaneous coronary intervention (PCI) when appropriate, further blood samples were obtained at six consecutive timepoints during hospitalisation (day 1: evening, day 2: morning, afternoon, evening, day 3: morning, afternoon). Blood samples were also obtained at 3 and 6 months follow-up.

Details on randomisation, treatment allocation, drug administration and pharmacology are reported elsewhere.[7]

\section{Blood sampling protocol}


Peripheral venous blood was drawn into endotoxin free blood collection tubes with EDTA as anticoagulant (plasma) and with no additives (serum). The EDTA tubes were immediately placed on melting ice and centrifuged within 30 minutes at $>2500 \times \mathrm{g}$ for 20 minutes to obtain platelet-poor plasma. Serum was centrifuged at $>2100 \times \mathrm{g}$ for 15 minutes after full coagulation ( 45 minutes) in room temperature. Immediately following centrifugation, both plasma and serum aliquots were stored at $-80^{\circ} \mathrm{C}$ until analyses in multiple aliquots. Samples were thawed only once.

\section{Biochemical analyses}

Components of the cytokine network were analysed in plasma according to the manufacturer's protocol using a multiplex cytokine assay (Bio-Plex Pro ${ }^{\mathrm{TM}}$ Human Cytokine Plex-27 Assay, Bio-Rad Laboratories, Hercules, CA) analyzing IL-1 $\beta$, IL-1 receptor antagonist (IL-1ra), IL-2, IL-4, IL-5, IL-6, IL-7, IL-8/CXCL-8, IL-9, IL-10, IL-12 (p70), IL13, IL-15, IL-17, basic fibroblast growth factor (bFGF), granulocyte colony-stimulating factor (G-CSF), granulocyte-macrophage colony-stimulating factor (GM-CSF), interferon-gamma

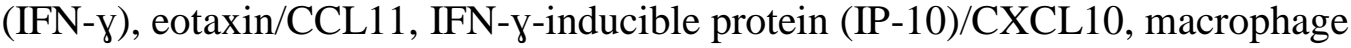
chemoattractant protein (MCP-1)/CCL2, macrophage inflammatory protein-1 $\alpha$ (MIP$1 \alpha /$ CCL3), MIP-1 $\beta /$ CCL4, regulated on activation, normal T-cell expressed and secreted (RANTES)/CCL5, tumor necrosis factor (TNF), platelet-derived growth factor (PDGF), and vascular endothelial growth factor (VEGF). Inter-assay and intra-assay co-efficient of variations were $<15$ and $<11$ for all analytes, respectively.

High-sensitivity C-reactive protein (hsCRP) was analysed on a MODULAR platform (Roche Diagnostics, Basel, Switzerland), and high-sensitivity troponin T (hsTnT) was measured by electrochemiluminescence immunoassay (ELICA; Elecsys 2010 analyzer, Roche Diagnostics). Leukocytes with subgroups were analysed consecutively using routine methods at the hospitals' clinical biochemistry laboratories. 


\section{Endpoints}

The main endpoint was to evaluate any differential effects of tocilizumab treatment on 27 different components of the cytokine network in patients with acute NSTEMI during hospitalisation, by the presence of a significant tocilizumab*time interaction. Secondary endpoints were differential effects of tocilizumab treatment on components of the cytokine network at 3 and 6 months follow-up, and if there were any within-group changes in levels at follow-up visits compared to baseline. We also evaluated associations between cytokines significantly affected by tocilizumab treatment and hsCRP, hsTnT and IL-6.

\section{Statistics}

The statistical analyses were performed with a per-protocol approach. IL-2, IL-15 and PDGF had in total (of all values in 117 patients over all timepoints) $>10 \%$ values out-oflower-range (OOR) and were left out of further statistical analyses. For IL-8, $15 \%$ of the samples had to be excluded due to technical problems with one of the plates, and analyses could unfortunately not be repeated. To avoid loss of low level data, values OOR and values $<1.0 \mathrm{pg} / \mathrm{ml}$ were set to $1.0 \mathrm{pg} / \mathrm{ml}$ for all cytokines included in further statistical analyses, except for IL1- $\beta$ for which values OOR and $<0.5 \mathrm{pg} / \mathrm{ml}$ were set to $0.5 \mathrm{pg} / \mathrm{ml}$. Further details regarding power estimation and statistical analyses are described in the Supplementary Material online. 


\section{Results}

\section{Baseline characteristics}

121 patients with NSTEMI were enrolled between August 2011 and November 2013. Follow-up ended in April 2014. Of these, 117 patients were included in the final analyses. There were no between-group differences in baseline characteristics (Table 1). Details regarding the screening and inclusion process, and dropout of patients (placebo $n=3$, tocilizumab n=3) are described previously.[7]

\section{Cytokine network during hospitalisation}

We have previously reported a significant increase during hospitalisation for IL-6 in the tocilizumab group for these patients,[7] as also replicated using the IL-6 multiplex assay in the current study (Figure 1, Table 2). Furthermore, we also observed a similar highly significant tocilizumab*time interaction for IP-10 and MIP-1 $\beta$ during hospitalisation (Figure 1, Table 2). Both cytokines were stable during hospitalisation in the placebo group, whereas a rapid and substantial increase was observed in the tocilizumab group. Furthermore, a more subtle, but significant tocilizumab*time interaction was observed for IL-8, reflecting a fall in the placebo group and no increase in the tocilizumab group (Figure 1, Table 2).

For the other evaluated cytokines no between-group significant impact of tocilizumab was observed (Table 2).

\section{Cytokine network at 3 and 6 months follow-up}

Except for a modest between-group difference for bFGF, there were no significant between-group differences in patterns of change from baseline for other cytokines at 3 and 6 months follow-up (Table 3). In both treatment groups, with a few exceptions, levels of cytokines were significantly lower at both 3 and 6 months follow-up compared to baseline. However, levels of IP-10 increased significantly compared to baseline in both groups at 3 months follow-up, whereas levels were comparable to baseline at 6 months. Also, in both 
groups, there were no significant changes in levels of MIP-1 $\beta$ compared to baseline at 3 and 6 months follow-up.

\section{Impact of revascularisation therapy on cytokine levels during hospitalisation}

The effects of tocilizumab on IP-10 and MIP-1 $\beta$, as well as previously described for IL-6,[7] were not affected by revascularisation therapy (PCI performed vs PCI not performed) (Supplementary Tables S1 and S2). However, while there was no between-group difference during hospitalisation for IL-8 in patients treated with PCI, a significant tocilizumab*time interaction was observed for IL-8 in patients not treated with PCI, reflecting a decrease in IL8 in the placebo group (Supplementary Table S2).

\section{Impact of time from symptom onset on cytokine levels during hospitalisation}

Also for patients included $\leq 2$ days (early) from symptom onset, the same betweengroup differences as for all patients were observed for IP-10 and MIP-1 $\beta$ as well as for IL-

6,[7] but not for IL-8 (Supplementary Table S3). However, in patients included >2 days (late) from symptom onset, equivalent significant between-group differences were observed for MIP-1 $\beta$ only, in addition to IL-6, but not for IL-8 or IP-10 (Supplementary Table S4).

\section{Correlations between IP-10, MIP-1ß and IL-6, hsCRP, leukocytes and hsTnT during hospitalisation}

We have previously shown that tocilizumab treatment caused a significant attenuation of CRP levels and PCI-related TnT release, as well as a substantial rise in IL-6 levels (reflecting attenuated elimination, but decreased IL-6 bioacitvity), and a profound decrease in neutrophils during hospitalisation in these patients.[7] There were no associations between area under the curve (AUC) for IL-6 and AUCs for IP-10 or MIP-1 $\beta$ in either treatment group during hospitalisation (Table 4). However, there was a significant positive association between AUCs for IP-10 and CRP in the placebo group, and significant inverse associations between AUCs for MIP-1 $\beta$ and AUCs for neutrophils and TnT (Table 4). 


\section{Discussion}

We have previously shown that IL-6 inhibition with tocilizumab attenuated inflammation and PCI-related TnT-release in patients with NSTEMI.[7] In this pre-defined sub-study, we explored the effects of tocilizumab on the cytokine network in these patients. Our main findings were; (i) Most cytokines remained unaffected by tocilizumab treatment. (ii) However, tocilizumab led to a profound and selective increase in IP-10 and MIP-1 $\beta$, whereas the effect on IL-8 was more complex. Overall, tocilizumab seemed to exert modest and selective effects on the universal inflammatory surge during the acute phase of NSTEMI, and the causes and implications of the elevated levels of especially IP-10 and MIP-1 $\beta$ requires further attention in forthcoming studies.

Whereas most analysed cytokines were elevated during hospitalisation as compared with follow-up, there was somewhat surprisingly no effect of tocilizumab on these mediators, except for IP-10, MIP-1 $\beta$ and IL-8. There are some in vitro studies showing that tocilizumab inhibits TNF[12] and other inflammatory cytokines,[13] and tocilizumab has been reported to down-regulate IL-21 in humans.[14] There is also one study showing that tocilizumab downregulates several inflammatory cytokines in patients with rheumatoid arthritis (ref), but this study had no placebo group. The present study is, however, to the best of our knowledge, the first report on the effect of tocilizumab on the cytokine network in a placebo-controlled randomised trial in humans. It has been claimed that IL-6 functions primarily as a secondary signaling cytokine, with a rather narrow effect on the cytokine network (ref), and the present study could support such a notion. However, tocilizumab markedly down-regulated CRP in patients in the present study.[7]

In contrast to most of the examined cytokines, tocilizumab had a significant effect on IP-10 and MIP-1 $\beta$, with increasing levels of both cytokines during IL-6R blockade. The potential consequences of these effects, if any, is at present unclear, but some possibilities 
exist. IP-10 is a chemokine which is known to be upregulated during MI.[15, 16] A role of IP10 in I/R-injury in MI has been suggested, possibly related to effects on leukocyte subpopulations.[15] However, previous studies have so far reported conflicting results regarding IP-10's association with infarct size in patients with reperfused MI, $[15,16]$ and there were no associations between IP-10 and TnT levels during hospitalisation in either of the groups in the present study. Thus, the current body of evidence does not provide compelling evidence that IP-10 holds a central position in the inflammatory arm of I/R-injury, and the potentially cardioprotective effect of IL-6 inhibition in patients with NSTEMI in the present study,[7] does not appear to be mediated via this chemokine. However, in an experimental study on mice undergoing myocardial I/R protocols, IP-10 deficient mice experienced accentuated LV remodeling compared to controls, which seem contradictory to the notion that suppression of IP-10 is essential to counteract LV remodeling following MI.[17] Thus, neither previous data nor the present study provide convincing evidence that high levels of IP-10 are either beneficial or clearly harmful during MI.

MIP-1 $\beta$ is a chemokine which seems to be upregulated in MI and to be involved in leukocyte recruitment to the infarcted myocardium.[18] Previously, levels of MIP-1 $\beta$ has been shown to be positively correlated to infarct size in patients with reperfused MI, which in turn could indicate an unfavourable role in I/R injury.[15] On the other hand, in a rat myocardial I/R-model, MIP-1 $\beta$ and signaling through on of its receptors CCR5, seemed to restrain postinfarction inflammation and the adverse LV remodeling through increased myocardial recruitement of T regulatory cells.[18] Herein, MIP-1 $\beta$ was substantially increased during hospitalisation in patients treated with tocilizumab. We have previously reported that tocilizumab caused a profound decrease in neutrophil counts during hospitalisation.[7] This phenomenon may be involved in the potentially cardioprotective effect of IL-6 inhibition reflected by attenuated TnT release in these patients.[7] Interestingly, 
MIP-1 $\beta$ was inversely correlated to both TnT and neutrophils in the tocilizumab group. Thus, the inverse correlation between elevated levels of MIP-1 $\beta$ and neutrophils in tocilizumab treated patients, may suggest that MIP-1 $\beta$ is involved in tocilizumab's suppressive and potentially beneficial effect on neutrophil counts. However, it is also possible that the inverse correlation between MIP-1 $\beta$ and neutrophils could reflect that this chemokine is directing the leukocytes into the myocardium, away from the circulation, and thereby mediating harmful effects.

This study has some limitations. The conspicuously absent effect of IL-6 inhibition on the majority of components in the cytokine network, might be due to the wide distribution in time from symptom onset to inclusion in this study, as patients could have been included at a time when the primary myocardial injury already had occurred. Furthermore, plasma levels do not necessarily reflect cytokine levels at the site of inflammation and it is possible that tocilizumab could have a broader effect within the myocardium and the coronary plaque. Finally, associations do not prove any causal relationship and more mechanistic studies are needed to elucidate the effects of IL-6 inhibition in NSTEMI.

Inflammation plays a crucial role in the pathogenesis of atherosclerosis, plaque rupture and MI, and there is an urgent need for treatment modalities that are targeting the inflammatory arm of atherogenesis, I/R-injury and cardiac repair mechanisms after MI. This study shows that a single dose of the IL-6R antagonist tocilizumab selectively increased IP-10 and MIP-1 $\beta$ levels in NSTEMI patients during hospitalisation, with no effects on the other cytokines. Further studies are needed to clarify the role of IP-10 and MIP-1 $\beta$ during NSTEMI, and forthcoming studies should also address through what mechanisms tocilizumab attenuates TnT release in these patients. The limited impact of IL-6 inhibition on the cytokine network in these patients, could suggest that the beneficial effects may be caused by effects directly 
related to IL-6, or mediated through CRP, possibly underscoring the pivotal role of this cytokine in post-MI inflammation.

\section{Acknowledgements}

This study was financially supported by The Norwegian Council on Cardiovascular Disease, The Odd Fellow Foundation, and the European Community's Seventh Framework Programme under grant agreement $n^{\circ} 602699$ (DIREKT) (TEM). 


\section{References}

[1] Libby P, Ridker PM, Hansson GK. Inflammation in atherosclerosis: from pathophysiology to practice. Journal of the American College of Cardiology. 2009;54:2129-38.

[2] Frangogiannis NG. The inflammatory response in myocardial injury, repair, and remodelling. Nature reviews Cardiology. 2014;11:255-65.

[3] Yellon DM, Hausenloy DJ. Myocardial reperfusion injury. The New England journal of medicine. 2007;357:1121-35.

[4] Yudkin JS, Kumari M, Humphries SE, Mohamed-Ali V. Inflammation, obesity, stress and coronary heart disease: is interleukin-6 the link? Atherosclerosis. 2000;148:209-14.

[5] Zamani P, Schwartz GG, Olsson AG, Rifai N, Bao W, Libby P, et al. Inflammatory biomarkers, death, and recurrent nonfatal coronary events after an acute coronary syndrome in the MIRACL study. Journal of the American Heart Association. 2013;2:e003103.

[6] Sawa Y, Ichikawa H, Kagisaki K, Ohata T, Matsuda H. Interleukin-6 derived from hypoxic myocytes promotes neutrophil-mediated reperfusion injury in myocardium. The Journal of thoracic and cardiovascular surgery. 1998;116:511-7.

[7] Kleveland O, Kunszt G, Bratlie M, Ueland T, Broch K, Holte E, et al. Effect of a single dose of the interleukin- 6 receptor antagonist tocilizumab on inflammation and troponin $\mathrm{T}$ release in patients with non-ST-elevation myocardial infarction: a double-blind, randomized, placebo-controlled phase 2 trial. European heart journal. 2016;37:2406-13.

[8] Ohtsuka T, Hamada M, Inoue K, Ohshima K, Sujzuki J, Matsunaka T, et al. Relation of circulating interleukin-6 to left ventricular remodeling in patients with reperfused anterior myocardial infarction. Clinical cardiology. 2004;27:417-20.

[9] Guillen I, Blanes M, Gomez-Lechon MJ, Castell JV. Cytokine signaling during myocardial infarction: sequential appearance of IL-1 beta and IL-6. The American journal of physiology. 1995;269:R229-35. 
[10] Castell JV, Gomez-Lechon MJ, David M, Andus T, Geiger T, Trullenque R, et al. Interleukin-6 is the major regulator of acute phase protein synthesis in adult human hepatocytes. FEBS letters. 1989;242:237-9.

[11] Smolen JS, Beaulieu A, Rubbert-Roth A, Ramos-Remus C, Rovensky J, Alecock E, et al. Effect of interleukin-6 receptor inhibition with tocilizumab in patients with rheumatoid arthritis (OPTION study): a double-blind, placebo-controlled, randomised trial. Lancet (London, England). 2008;371:987-97.

[12] Shin BH, Ge S, Mirocha J, Jordan SC, Toyoda M. Tocilizumab (Anti-IL-6R) Suppressed TNFalpha Production by Human Monocytes in an In Vitro Model of Anti-HLA AntibodyInduced Antibody-Dependent Cellular Cytotoxicity. Transplantation direct. 2017;3:e139.

[13] Mizwicki MT, Fiala M, Magpantay L, Aziz N, Sayre J, Liu G, et al. Tocilizumab attenuates inflammation in ALS patients through inhibition of IL6 receptor signaling. American journal of neurodegenerative disease. 2012;1:305-15.

[14] Carbone G, Wilson A, Diehl SA, Bunn J, Cooper SM, Rincon M. Interleukin-6 receptor blockade selectively reduces IL-21 production by CD4 $\mathrm{T}$ cells and IgG4 autoantibodies in rheumatoid arthritis. International journal of biological sciences. 2013;9:279-88.

[15] Orn S, Breland UM, Mollnes TE, Manhenke C, Dickstein K, Aukrust P, et al. The chemokine network in relation to infarct size and left ventricular remodeling following acute myocardial infarction. The American journal of cardiology. 2009;104:1179-83.

[16] Koten K, Hirohata S, Miyoshi T, Ogawa H, Usui S, Shinohata R, et al. Serum interferongamma-inducible protein 10 level was increased in myocardial infarction patients, and negatively correlated with infarct size. Clinical biochemistry. 2008;41:30-7.

[17] Bujak M, Dobaczewski M, Gonzalez-Quesada C, Xia Y, Leucker T, Zymek P, et al. Induction of the CXC chemokine interferon-gamma-inducible protein 10 regulates the reparative response following myocardial infarction. Circulation research. 2009;105:973-83. 
[18] Dobaczewski M, Xia Y, Bujak M, Gonzalez-Quesada C, Frangogiannis NG. CCR5 signaling suppresses inflammation and reduces adverse remodeling of the infarcted heart, mediating recruitment of regulatory $\mathrm{T}$ cells. The American journal of pathology. 2010;176:2177-87. 


\begin{tabular}{|c|c|c|c|}
\hline & Placebo $(n=59)$ & Tocilizumab $(\mathrm{n}=58)$ & $P$-Value \\
\hline Age, y, mean (SD) & $60.1(9.9)$ & $59.8(7.7)$ & 0.859 \\
\hline Female, n (\%) & $5(8.5)$ & $9(15.5)$ & 0.364 \\
\hline Body mass index, $\mathrm{kg} / \mathrm{m}^{2}$, mean (SD) & $27.4(4.4)$ & $28.8(3.3)$ & 0.055 \\
\hline Blood pressure, systolic, mm Hg, mean (SD) & $136.8(18.0)$ & $139.7(18.1)$ & 0.389 \\
\hline Blood pressure, diastolic, mmHg, mean (SD) & $80.5(12.1)$ & $82.9(12.0)$ & 0.273 \\
\hline Hypertension, n (\%) & $17(28.8)$ & $26(44.8)$ & 0.109 \\
\hline Diabetes Mellitus, n (\%) & $10(16.9)$ & $11(19.0)$ & 0.966 \\
\hline Current Smoking, n (\%) & $17(28.8)$ & $15(26.3)$ & 0.926 \\
\hline Previous Myocardial Infarction, n (\%) & $7(11.9)$ & $9(15.5)$ & 0.760 \\
\hline GRACE score, mean (SD) & $90.3(20.2)$ & $86.3(19.1)$ & 0.272 \\
\hline $\begin{array}{l}\text { Symptom onset to inclusion, days, median } \\
\text { (25th, } 75 \text { th percentiles) }\end{array}$ & $2(1,3)$ & $2(1,3.5)$ & 0.197 \\
\hline Maximum TnT before baseline, ng/L, n=85, geometric mean $(\mathrm{CI})$ & $310(214-449)$ & $334(217-514)$ & 0.789 \\
\hline Maximum $\mathrm{TnI}$ before baseline, $\mathrm{ng} / \mathrm{L}, \mathrm{n}=31$, geometric mean $(\mathrm{CI})$ & $1571(517-4773)$ & $2221(826-5976)$ & 0.626 \\
\hline Aspirin, $\mathrm{n}(\%)$ & $59(100)$ & $57(98.3)$ & 0.496 \\
\hline Clopidogrel, n (\%) & $32(54.2)$ & $32(55.2)$ & 1.0 \\
\hline Ticagrelor, n (\%) & $27(45.8)$ & $26(44.8)$ & 1.0 \\
\hline Low molecular weight heparin, $\mathrm{n}(\%)$ & $54(91.5)$ & $51(89.5)$ & 0.952 \\
\hline Statin, n (\%) & $53(89.8)$ & $53(91.4)$ & 1.0 \\
\hline Betablocker, n (\%) & $45(76.3)$ & 45 (77.6) & 1.0 \\
\hline PCI, n (\%) & $47(79.7)$ & $41(70.7)$ & 0.367 \\
\hline Stents per PCI-treated patient, mean (SD) & $1.85(1.28)$ & $1.85(1.15)$ & 0.982 \\
\hline CABG, n (\%) & 7 (11.9) & $6(10.3)$ & 1.0 \\
\hline Medical treatment, n (\%) & $5(8.5)$ & $11(19.0)$ & 0.167 \\
\hline $\begin{array}{l}\text { SYNTAX score, median } \\
\text { (25th, } 75 \text { th percentiles) }\end{array}$ & $8.0(5,14)$ & $8.0(4,18)$ & 0.629 \\
\hline
\end{tabular}

TnT, troponin T; TnI, troponin I; PCI, percutaneous coronary intervention; CABG, Coronary Artery Bypass Grafting 
Table 2. Multiplex cytokine assay in patients with non-ST-elevation myocardial infarction receiving placebo $(\mathrm{n}=59)$ and tocilizumab $(\mathrm{n}=58)$.

\begin{tabular}{|c|c|c|c|c|c|c|c|c|c|}
\hline $\begin{array}{l}\text { Cytokine } \\
(\mathrm{pg}(\mathrm{mL})\end{array}$ & Group & Baseline & Evening day 1 & Morning day 2 & Afternoon day2 & Evening day 2 & Morning day 3 & Afternoon day 3 & $\begin{array}{c}\text { Repeated } \\
\text { measures, } P \dagger\end{array}$ \\
\hline \multirow{2}{*}{ IL-1 $\beta$} & Placebo & $5.8(2.9,9.7)$ & $3.0(1.3,5.1)$ & $5.9(2.8,8.1)$ & $4.3(2.0,7.2)$ & $3.5(1.3,5.7)$ & $3.9(2.2,7.5)$ & $3.5(2.1,5.7)$ & \multirow{2}{*}{0.151} \\
\hline & Tocilizumab & $4.8(2.0,10.2)$ & $4.2(1.6,6.1)$ & $4.3(2.2,8.0)$ & $4.1(2.1,6.8)$ & $4.1(1.6,7.0)$ & $4.8(2.1,8.2)$ & $3.3(1.8,6.7)$ & \\
\hline \multirow{2}{*}{ IL-1ra } & Placebo & $113(73-176)$ & $76(52-111)$ & $115(86-155)$ & 99 (71-139) & $80(55-116)$ & $82(57-118)$ & $85(60-122)$ & \multirow[b]{2}{*}{0.657} \\
\hline & Tocilizumab & $147(103-210)$ & $98(71-134)$ & $112(85-148)$ & $109(83-143)$ & $104(75-144)$ & $94(69-129)$ & $98(73-130)$ & \\
\hline \multirow{2}{*}{ IL-4 } & Placebo & $4.4(2.4,6.2)$ & $2.8(1.0,4.7)$ & $4.1(2.2,5.3)$ & $3.4(2.2,5.1)$ & $3.0(1.2,4.7)$ & $3.1(1.9,4.9)$ & $3.2(1.7,4.6)$ & \multirow{2}{*}{0.353} \\
\hline & Tocilizumab & $4.1(2.1,6.6)$ & $3.3(1.7,4.7)$ & $3.3(2.0,5.5)$ & $3.6(2.0,4.9)$ & $3.3(1.8,4.8)$ & $2.8(2.1,5.1)$ & $3.1(1.7,4.7)$ & \\
\hline \multirow{2}{*}{ IL-5 } & Placebo & $21(12,29)$ & $14(7,21)$ & $19(12-25)$ & $17(10-23)$ & $14(8-21)$ & $14(8-22)$ & $13(10-22)$ & \multirow{2}{*}{0.588} \\
\hline & Tocilizumab & $19(11-29)$ & $14(8-22)$ & $16(9-23)$ & $16(9-22)$ & $15(7-24)$ & $16(9-20)$ & $13(7-20)$ & \\
\hline \multirow{2}{*}{ IL-6 } & Placebo & $8(6-10)$ & $10(8-12)^{*}$ & $10(9-13)^{* * *}$ & $9(8-11)$ & $8(6-9)$ & $8(6-9)$ & $6(5-8) *$ & \multirow{2}{*}{$<0.001$} \\
\hline & Tocilizumab & $7(6-9)$ & $37(29-46) * * * \mathrm{C}$ & $40(33-50)^{* * * \mathrm{C}}$ & $34(28-41) * * * \mathrm{C}$ & $36(30-44) * * * \mathbf{c}$ & $37(30-45) * * * \mathrm{C}$ & $31(25-39) * * * \mathrm{C}$ & \\
\hline \multirow{2}{*}{ IL-7 } & Placebo & $26(21-33)$ & $17(14-21)$ & $24(20-30)$ & $22(17-27)$ & $16(13-20)$ & $22(18-28)$ & $19(15-23)$ & \multirow{2}{*}{0.530} \\
\hline & Tocilizumab & $27(20-34)$ & $19(15-24)$ & $27(22-32)$ & $20(15-26)$ & $18(15-23)$ & $23(18-30)$ & $22(17-27)$ & \\
\hline \multirow{2}{*}{ IL-8 } & Placebo & $16(10,30)$ & $11(7,20) * * *$ & $17(10,26)$ & $14(10,22) * *$ & $11(7,17) * * *$ & $13(8,24)^{*}$ & $12(8,20) * * *$ & \multirow{2}{*}{0.019} \\
\hline & Tocilizumab & $13(9,32)$ & $15(10,22)$ & $16(11,25)$ & $13(9,22)$ & $13(9,21)$ & $15(10,25)$ & $13(10,22)$ & \\
\hline \multirow{2}{*}{ IL-9 } & Placebo & $16(12-22)$ & $10(8-13)$ & $16(13-21)$ & $14(11-18)$ & $10(7-13)$ & $14(10-19)$ & $11(8-15)$ & \multirow[b]{2}{*}{0.303} \\
\hline & Tocilizumab & $18(13-25)$ & $12(9-17)$ & $18(14-23)$ & $14(10-19)$ & $13(10-17)$ & $15(11-21)$ & $15(11-20)$ & \\
\hline \multirow{2}{*}{ IL-10 } & Placebo & $21(15-30)$ & $11(8-16)$ & $20(15-27)$ & $16(12-23)$ & $12(8-16)$ & $16(12-23)$ & $13(9-19)$ & \multirow[b]{2}{*}{0.278} \\
\hline & Tocilizumab & $22(15-33)$ & $13(9-18)$ & $17(12-23)$ & $13(9-19)$ & $11(8-15)$ & $15(11-21)$ & $14(10-20)$ & \\
\hline \multirow{2}{*}{ IL-12 } & Placebo & $33(24-46)$ & $18(13-24)$ & $31(24-41)$ & $25(19-34)$ & $18(13-25)$ & $26(18-37)$ & $21(15-28)$ & \multirow{2}{*}{0.343} \\
\hline & Tocilizumab & $32(23-45)$ & $19(14-26)$ & $27(21-35)$ & $21(16-29)$ & $18(13-24)$ & $24(18-33)$ & $23(17-32)$ & \\
\hline & Placebo & $22(16-30)$ & $10(7-15)$ & $17(13-23)$ & $14(10-20)$ & $10(7-14)$ & $14(10-19)$ & $12(9-17)$ & \\
\hline $1 \mathrm{~L}-13$ & Tocilizumab & $22(16-30)$ & $12(9-17)$ & $16(13-21)$ & $15(11-19)$ & $12(9-15)$ & $14(11-19)$ & $15(12-20)$ & 0.407 \\
\hline & Placebo & $39(21,68)$ & $24(8,44)$ & $38(20,61)$ & $29(14,57)$ & $27(11,50)$ & $33(12,58)$ & $28(13,46)$ & \\
\hline IL-17 & Tocilizumab & $42(18,80)$ & $31(18,57)$ & $39(19,65)$ & $39(20,60)$ & $35(19,52)$ & $37(18,64)$ & $34(17,58)$ & 0.204 \\
\hline & Placebo & $91(66,127)$ & $78(56,104)$ & $86(68,120)$ & $95(64,115)$ & $77(59,100)$ & $83(57,109)$ & $75(58,111)$ & \\
\hline Eotaxin & Tocilizumab & $100(59,157)$ & $84(56,135)$ & $83(62,169)$ & $87(59,168)$ & $84(59,151)$ & $87(59,137)$ & $90(61,147)$ & 0.299 \\
\hline & Placebo & $64(41,87)$ & $45(24,62)$ & $64(37,82)$ & $54(32,74)$ & $49(28,73)$ & $51(32,76)$ & $48(32,70)$ & 0684 \\
\hline Bas FGF & Tocilizumab & $63(36,93)$ & $48(31,66)$ & $54(32,80)$ & $57(29,77)$ & $51(29,69)$ & $50(30,78)$ & $52(29,78)$ & 0.684 \\
\hline & Placebo & $58(45-73)$ & $39(31-49)$ & $55(45-66)$ & $43(32-57)$ & $36(27-49)$ & $42(32-55)$ & $46(37-56)$ & \\
\hline G-cst & Tocilizumab & $61(50-74)$ & $46(37-57)$ & $54(45-46)$ & $50(42-61)$ & $48(40-59)$ & $49(40-61)$ & $51(42-63)$ & 0.322 \\
\hline & Placebo & $57(25,67)$ & $39(18,54)$ & $49(28,68)$ & $45(24,63)$ & $39(18,59)$ & $35(23,63)$ & $40(20,59)$ & \\
\hline GM-csf & Tocilizumab & $51(20,82)$ & $40(19,65)$ & $41(22,66)$ & $43(22,63)$ & $40(18,65)$ & $42(18,67)$ & $41(18,58)$ & 0.403 \\
\hline 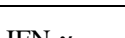 & Placebo & $162(83,232)$ & $100(33,168)$ & $161(75,210)$ & $119(77,199)$ & $111(36,184)$ & $116(57,190)$ & $116(64,170)$ & 0.957 \\
\hline $\mathrm{IFN}-\gamma$ & Tocilizumab & $138(69,261)$ & $114(66,182)$ & $133(60,211)$ & $124(68,198)$ & $121(54,176)$ & $112(64,216)$ & $117(59,197)$ & $0.85 /$ \\
\hline & Placebo & $381(275,672)$ & $366(252,561)^{* *}$ & $495(286,783)$ & $431(257,694)$ & $433(266,664)$ & $481(349,741)^{* *}$ & $447(305,757)$ & \\
\hline IP-10 & Tocilizumab & $421(282,899)$ & $630(418,954)^{* * * \mathrm{C}}$ & $754(477,1289) * * * \mathbf{C}$ & $613(450,947)^{* * * \mathrm{C}}$ & $587(429,892) * * * \mathrm{C}$ & $718(487,1155)^{* * * \mathrm{C}}$ & $556(384,961) * * * \mathrm{C}$ & $<0.001$ \\
\hline & Placebo & $40(25,73)$ & $30(17,47)$ & $41(24,65)$ & $37(22,59)$ & $28(17,53)$ & $33(20,60)$ & $29(18,51)$ & \\
\hline MCP-1 & Tocilizumab & $40(20,83)$ & $35(20,60)$ & $37(21,65)$ & $35(20,64)$ & $36(17,53)$ & $36(23,65)$ & $31(17,61)$ & 0.265 \\
\hline & Placebo & $85(47,131)$ & $57(29,85)$ & $86(50,114)$ & $70(40,104)$ & $58(29,98)$ & $74(43,114)$ & $55(37,98)$ & \\
\hline MIP-1 $\alpha$ & Tocilizumab & $77(47,135)$ & $63(39,100)$ & $72(44,110)$ & $70(37,92)$ & $58(39,84)$ & $74(40,113)$ & $64(41,101)$ & 0.491 \\
\hline & Placebo & $61(54-68)$ & $61(55-68)$ & $69(62-77)^{* * *}$ & $64(57-72)$ & $63(57-71)$ & $75(67-83)^{* * *}$ & $65(58-72)$ & \\
\hline MIP-1 $\beta$ & Tocilizumab & $59(54-65)$ & $97(85-110)^{* * * \mathrm{C}}$ & $113(101-126)^{* * * \mathrm{C}}$ & $96(86-107)^{* * * \mathrm{C}}$ & $90(81-100)^{* * * \mathrm{C}}$ & $108(97-120)^{* * * \mathrm{C}}$ & $88(79-98) * * * \mathrm{C}$ & $<0.001$ \\
\hline & Placebo & $451(213,1231)$ & $551(195,1385)$ & $570(182,1300)$ & $677(197,1143)$ & $406(216,1697)$ & $393(164,1008)$ & $610(285,1272)$ & 0900 \\
\hline RANIES & Tocilizumab & $418(249,1161)$ & $411(196,896)$ & $366(211,881)$ & $470(181,1203)$ & $437(148,2201)$ & $352(162,818)$ & $386(198,1298)$ & 0.900 \\
\hline TNE & Placebo & $60(21,78)$ & $27(8,51)$ & $49(16,65)$ & $37(15,61)$ & $29(8,60)$ & $29(15,67)$ & $31(12,57)$ & הרר ח \\
\hline $1 N F-\alpha$ & Tocilizumab & $46(16,95)$ & $29(15,59)$ & $31(15,70)$ & $31(15,65)$ & $33(13,60)$ & $32(14,69)$ & $29(13,59)$ & 0.220 \\
\hline & Placebo & $23(16-34)$ & $14(10-19)$ & $23(17-31)$ & $19(14-26)$ & $14(10-20)$ & $18(13-26)$ & $15(11-21)$ & 0452 \\
\hline VEGF & Tocilizumab & $24(17-35)$ & $15(10-21)$ & $19(14-26)$ & $17(12-24)$ & $15(11-21)$ & $18(13-25)$ & $18(13-25)$ & 0.452 \\
\hline
\end{tabular}

Data are geometric mean and $\left(95 \%\right.$ confidence interval) and median $\left(25^{\text {th }}, 75^{\text {th }}\right.$ percentiles). ${ }^{*} p<0.05, * * p<0.01, * * * p<0.001$ vs baseline. ${ }^{C} p<0.001$ changes from baseline vs placebo same timepoint.

tinteraction tocilizumab*time in mixed between-within subjects analysis of variance during hospitalisation. 
Table 3. Multiplex cytokine assay in patients with non-ST-elevation myocardial infarction at baseline and during follow-up receiving placebo (n=59) and tocilizumab (n=58).

\begin{tabular}{|c|c|c|c|c|c|}
\hline Cytokine (pg/mL) & Group & Baseline & 3 months & 6 months & Repeated measures, $P \dagger$ \\
\hline \multirow{2}{*}{ IL-1 $\beta$} & Placebo & $5.8(2.6,9.7)$ & $2.9(1.3,4.3)^{* * * *}$ & $2,8(0.7,4.7)^{* * * *}$ & \multirow{2}{*}{0.617} \\
\hline & Tocilizumab & $4.8(1.9,10.3)$ & $3.0(1.2,5.0)^{* * *}$ & $3.7(1.1,4.5)^{* * *}$ & \\
\hline \multirow{2}{*}{ IL-1ra } & Placebo & $119(77-186)$ & $72(48-108)$ & $78(47-130)$ & \multirow{2}{*}{0.763} \\
\hline & Tocilizumab & $146(103-205)$ & $72(46-114) * * *$ & $95(61-148)$ & \\
\hline \multirow{2}{*}{ IL-4 } & Placebo & $4.5(2.4,6.3)$ & $2.7(1.0,4.0)^{* * * *}$ & $2.3(1.0,4.2)^{* * *}$ & \multirow{2}{*}{0.829} \\
\hline & Tocilizumab & $4.0(2.1,6.5)$ & $2.7(1.0,4.0)^{* * *}$ & $2.6(1.0,4.1)^{* * *}$ & \\
\hline \multirow{2}{*}{ IL-5 } & Placebo & $21(12,29)$ & $14(6,22) * * *$ & $11(4,23)^{* *}$ & \multirow[b]{2}{*}{0.614} \\
\hline & Tocilizumab & $20(11,30)$ & $13(4,23)^{* * *}$ & $12(4,24)^{* * *}$ & \\
\hline \multirow{2}{*}{ IL-6 } & Placebo & $7.7(6.9,9.8)$ & $3.2(2.6-3.9) * * *$ & $4.3(3.4-5.4)^{* * * *}$ & \multirow{2}{*}{0.878} \\
\hline & Tocilizumab & $7.5(5.9-9.5)$ & $3.4(2.7-4.2) * * *$ & $4.5(3.6-5.6) * *$ & \\
\hline \multirow{2}{*}{ IL-7 } & Placebo & $26(21-32)$ & $11(9-14)^{* * * *}$ & $10(8-13)^{* * * *}$ & \multirow[b]{2}{*}{0.744} \\
\hline & Tocilizumab & $26(20-33)$ & $13(10-16)^{* * * *}$ & $11(8-14)^{* * *}$ & \\
\hline \multirow{2}{*}{ IL-8 } & Placebo & $15(10,30)$ & $9(6,13)^{* * * *}$ & $9(6,11)^{* * * *}$ & \multirow{2}{*}{0.763} \\
\hline & Tocilizumab & $13(9,30)$ & $8(6,12) * * *$ & $8(6,10) * * *$ & \\
\hline \multirow[b]{2}{*}{ IL-9 } & Placebo & $16(12-22)$ & $7(6-10) * * *$ & $6(5-9)$ & \multirow[b]{2}{*}{0.969} \\
\hline & Tocilizumab & $19(22-26)$ & $8(6-12)^{* * *}$ & $7(5-10) * * *$ & \\
\hline \multirow[b]{2}{*}{ IL-10 } & Placebo & $20(14-29)$ & $9(6-12)^{* * * *}$ & $7(5-9)^{* * *}$ & \multirow{2}{*}{0.784} \\
\hline & Tocilizumab & $21(15-31)$ & $8(6-12) * * *$ & $7(5-10)$ & \\
\hline \multirow{2}{*}{ IL-12 } & Placebo & $32(22-45)$ & $15(11-20)^{* * * *}$ & $12(9-15) * * *$ & \multirow{2}{*}{0.580} \\
\hline & Tocilizumab & $31(22-43)$ & $13(9-17)^{* * * *}$ & $11(9-15)^{* * * *}$ & \\
\hline \multirow{2}{*}{ IL-13 } & Placebo & $22(16-30)$ & $8(6-10)^{* * * *}$ & $6(4-8)^{* * * *}$ & \multirow[b]{2}{*}{0.625} \\
\hline & Tocilizumab & $21(16-29)$ & $6(5-9) * * *$ & $6(5-8)^{* * *}$ & \\
\hline & Placebo & $38(21,69)$ & $25(9,37)^{* * *}$ & $22(7.36)^{* * * *}$ & \\
\hline $\mathrm{IL}-1 /$ & Tocilizumab & $42(18,76)$ & $24(11,41) * * *$ & $26(8,39) * * *$ & 0.454 \\
\hline & Placebo & $91(65,127)$ & $69(57,104)^{* * *}$ & $72(59,129)^{*}$ & \\
\hline Eotaxin & Tocilizumab & $96(59,152)$ & $71(57,124)^{* * *}$ & $78(59,137)$ & 0.884 \\
\hline & Placebo & $64(38,87)$ & $46(27,59)^{* * * *}$ & $38(20,55)^{* * *}$ & \\
\hline Bas FGF & Tocilizumab & $63(36,88)$ & $44(22,66) * *$ & $42(42,61) * * *$ & 0.034 \\
\hline & Placebo & $57(45-73)$ & $34(26-44)^{* *}$ & $32(25-42)^{* * *}$ & \\
\hline G-csf & Tocilizumab & $60(49-73)$ & $31(22-43) * * *$ & $36(28-48) * * *$ & 0.501 \\
\hline GM-csf & Placebo & $57(25,67)$ & $33(13,59)^{* * * *}$ & $33(13,57)^{* * * *}$ & 0607 \\
\hline GIM-cSI & Tocilizumab & $51(21,82)$ & $40(14,64)^{* *}$ & $39(19,60)^{* * *}$ & 0.001 \\
\hline & Placebo & $162(80,232)$ & $95(36,142)^{* * * *}$ & $107(34,163)^{* * * *}$ & \\
\hline $\mathrm{IFN}-\gamma$ & Tocilizumab & $138(69,261)$ & $104(25,149)^{* * *}$ & $116(28,162) * * *$ & 0.402 \\
\hline & Placebo & $381(273,672)$ & $464(318,872)^{* * * *}$ & $408(314,1044)$ & \\
\hline IP-10 & Tocilizumab & $420(276,783)$ & $456(351,772)^{* *}$ & $429(291,857)$ & 0.522 \\
\hline & Placebo & $40(24,73)$ & $23(13,37)^{* * * *}$ & $24(10,36)^{* * * *}$ & \\
\hline МСР-1 & Tocilizumab & $40(21,80)$ & $26(10,44) * * *$ & $26(13,43)^{* *}$ & 0.496 \\
\hline & Placebo & $83(42,131)$ & $44(33,64)^{* * * *}$ & $48(23,58)^{* * * *}$ & \\
\hline MIP-I $\alpha$ & Tocilizumab & $75(46,132)$ & $45(31,73) * * *$ & $42(29,61) * * *$ & 0.317 \\
\hline & Placebo & $61(54-68)$ & $58(52-64)$ & $59(53-66)$ & \\
\hline MIP-1 $\beta$ & Tocilizumab & $59(55-65)$ & $60(56-65)$ & $56(52-61)$ & 0.138 \\
\hline RANTES & Placebo & $460(262,1231)$ & $342(190,2137)$ & $491(166,2324)$ & \\
\hline RANIES & Tocilizumab & $460(261,1288)$ & $422(168,1916)$ & $629(196,2722)$ & 0.533 \\
\hline & Placebo & $60(21,78)$ & $23(8,55)^{* * *}$ & $31(12,65)^{*}$ & \\
\hline TNF- $\alpha$ & Tocilizumab & $46(16,95)$ & $25(7,61) * *$ & $36(10,69)^{* *}$ & 0.765 \\
\hline & Placebo & $23(16-33)$ & $11(8-15)^{* * *}$ & $10(7-14)^{* * * *}$ & \\
\hline VEGF & Tocilizumab & $23(16-33)$ & $12(9-16) * * *$ & $11(8-14) * * *$ & 0.862 \\
\hline
\end{tabular}

Data are geometric mean and (95\% confidence interval), median and $\left(25^{\text {th }}, 75^{\text {th }}\right.$ percentiles) and mean (standard deviation). ${ }^{*} \mathrm{p}<0.05,{ }^{* *} \mathrm{p}<0.01,{ }^{* * * *} \mathrm{p}<0.001$ vs baseline. ${ }^{\mathrm{A}} \mathrm{p}<0.05,{ }^{\mathrm{B}} \mathrm{p}<0.01,{ }^{\mathrm{C}_{\mathrm{p}}}<0.001 \mathrm{changes}$ from

baseline vs placebo same timepoint. finteraction tocilizumab*time in mixed between-within subjects analysis of variance during follow-up. 
Table 4. Pearson $r$ correlations between area under the curve during hospitalisation for IP-10, MIP-1 $\beta$ and IL-6, CRP, neutrophils and troponin T in patients with non-ST-elevation myocardial infarction. Placebo $n=59$, Tocilizumab $n=58$.

AUC IP-10

AUC MIP-1 $\beta$

\begin{tabular}{ccccc}
\hline & Placebo & Tocilizumab & Placebo & Tocilizumab \\
\hline AUC IL-6 & 0.168 & -0.129 & 0.205 & -0.111 \\
AUC CRP & $\mathbf{0 . 3 4 6 * *}$ & $-0,079$ & 0.061 & -0.158 \\
AUC Neutr & 0.208 & -0.155 & -0.078 & $\mathbf{- 0 . 3 2 1 *}$ \\
AUC TnT & 0.109 & -0.077 & -0.055 & $\mathbf{- 0 . 2 7 9 *}$ \\
\hline
\end{tabular}

${ }^{*} \mathrm{p}<0.05, * * \mathrm{p}<0.01$. IP-10, interferon gamma inducible protein/CXCL10. MIP-1 $\beta$, macrophage inflammatory protein-1 $\beta$. IL-6, interleukin-6. CRP, C-reactive protein. Neutr, neutrophils. TNT, troponin $\mathrm{T}$.
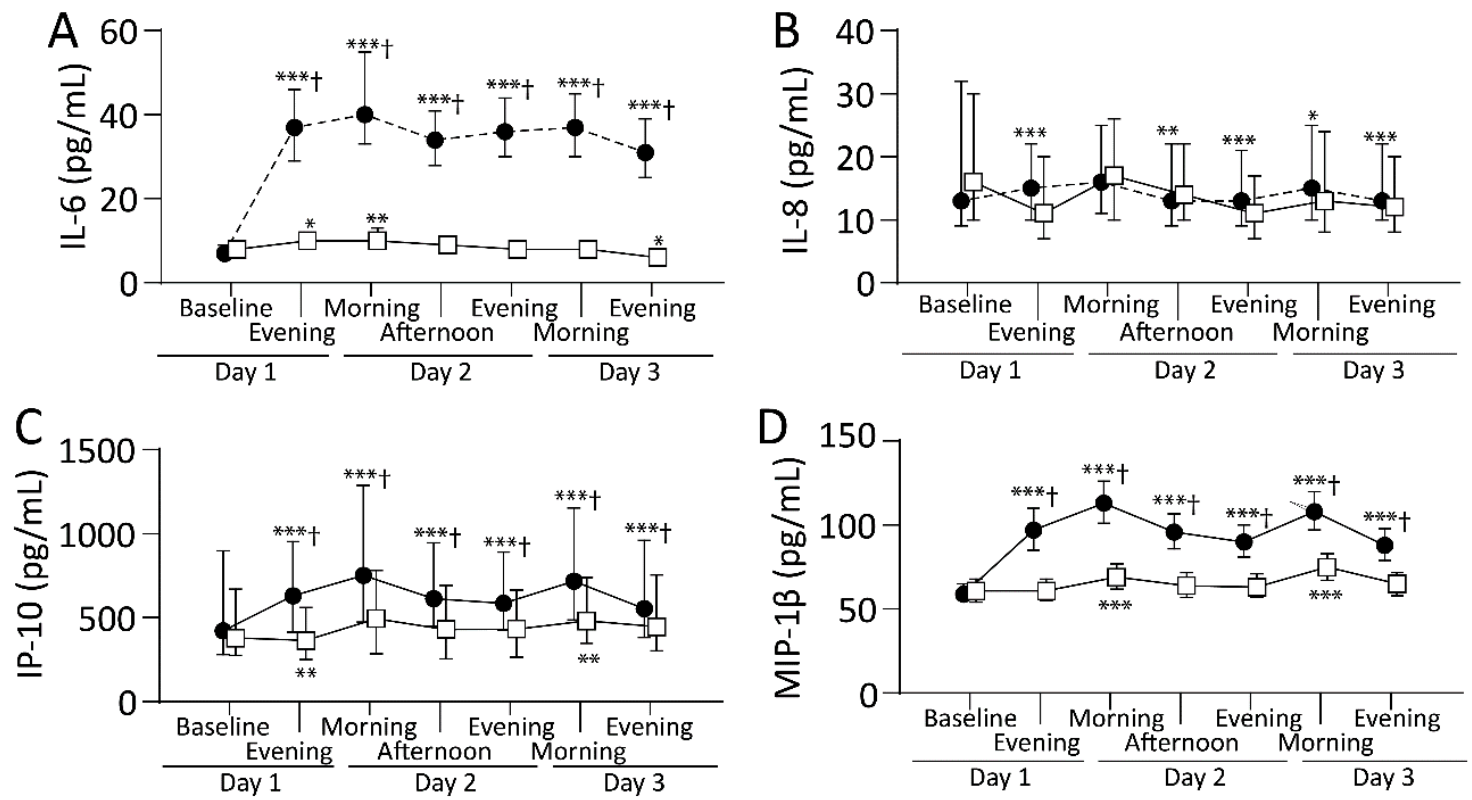
Figure 1. Interleukin-6 (IL-6), interferon gamma-inducible protein/CXCL10 (IP-10), macrophage inflammatory protein- $1 \beta$ (MIP-1 $\beta$ ) (all placebo $n=59$, tocilizumab $n=58$ ), and IL8 (placebo $n=49$, tocilizumab $n=46$ ) during hospitalisation in patients with non-ST-elevation myocardial infarction. Circles and squares represents geometric means (IL-6 and MIP-1 $\beta$ ) and median values (IL-8 and IP-10), bars represent $95 \%$ confidence intervals (IL-6 and MIP-1 $\beta$ ) and $25^{\text {th }}$ and $75^{\text {th }}$ percentiles (IL-8 and IP-10). ${ }^{*} \mathrm{p}<0.05, * * \mathrm{p}<0.01, * * * \mathrm{p}<0.001$ vs baseline within group. $\uparrow \mathrm{p}<0.001$ for between-group differences in changes from baseline at separate timepoints. 